\title{
MODERNISMO EN LA POESÍA DE MANUEL MACHADO: TRADICIÓN E INNOVACIÓN EN EL SONETO ALEJANDRINO
}

\author{
MODERNISM IN THE POETRY OF \\ MANUELMACHADO:TRADITIONANDINNOVATION \\ IN THE ALEXANDRIAN SONNET
}

\author{
Nora RodríGuez MarTíneZ \\ Universidad de Sevilla
}

Resumen: En este trabajo se estudia la renovación modernista que Manuel Machado desarrolla en su poética, especialmente el soneto alejandrino. En este sentido, se van a analizar las distintas innovaciones modernistas que el autor introduce en su poesía: las dislocaciones acentuales que dan ligereza y dinamismo al ritmo, la presencia o ausencia de palabras agudas o esdrújulas al final de hemistiquio que afectan al cómputo silábico, los encabalgamientos, la rima y los juegos fónicos, que buscan una poesía más expresiva y sugerente.

Palabras clave: Poesía, Modernismo, Manuel Machado, soneto alejandrino, innovaciones.

Abstract: This piece of work studies the modernist renewal developed by Manuel Machado in his poetry, especially the alexandrian sonnet. In this sense, it analyzes the 
different modernist innovations which the author introduces in his poetry: the accentual dislocations that give lightness and dynamism to the rhythm, the presence or absence of acute or esdrujula words at the end of hemistich affecting the syllabic computation, the syllable cross-overs, the rhyme and the phonics games, that seek a more expressive and evocative poetry.

Keywords: Poetry, Modernism, Manuel Machado, alexandrian sonnet, innovations. 


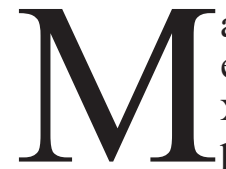

anuel Machado es una figura imprescindible para entender la evolución de la poesía española del siglo $\mathrm{xx}$, y ello, además, en un momento muy importante para las letras y el arte como es la encrucijada finisecular. Como afirma el propio Manuel Machado en su libro en prosa La guerra literaria:

La Poesía española se moría en medio del desprecio general. [...] Los libreros y editores repugnaban las colecciones de versos, rechazábanse estos en los periódicos, $\mathrm{y}$ en el Ateneo de Madrid, donde tan altas cosas se han dicho, pero donde casi toda necedad ha tenido también su asiento, se discutía en serio si la forma poética estaba llamada a desaparecer ${ }^{1}$.

De este modo, en vista de la situación, se hace evidente la necesidad de una renovación en poesía que ofrezca nuevas posibilidades de expresión al artista. Éste se siente cohibido por las normas establecidas y necesita recurrir a artificios nuevos para expresar nuevas ideas. En este sentido, el poeta andaluz llega a decir:

No me pago de purista, ni comprendo cómo hay quien se entretiene en eso, fuera de la Academia Española que tiene la obligación, y el gran Mariano de Cavia, que tiene el capricho. Con todo mi respeto a la Academia y mi devoción al maestro Cavia seguiré cometiendo galicismos siempre que me acomode para ser más expresivo y claro, es decir, siempre que me encuentre con que la expresión española está gastada y vieja, y no responde ya al matiz actual de una cosa o de una idea $^{2}$.

Hay que señalar, además, que, tal como afirma María Victoria Utrera en su libro Poéticas de la enfermedad en la literatura

${ }^{1}$ Machado, Manuel: La guerra literaria. Madrid: Narcea, 1981, pp. 103-104.

2 Ibid., p. 96. 
moderna, «el drama del artista moderno, es tener que someter su espíritu a un lenguaje previo e insuficiente, de ahí su propósito de inventar y encontrar un nuevo lenguaje»» ${ }^{3}$.

Así, poco a poco se fueron llevando a cabo diversas innovaciones en el campo de la poesía, hasta que el Modernismo penetró las fronteras de nuestro país, proceso en el que fue muy importante la labor de Manuel Machado como poeta. De hecho, él mismo comenta con mucho humor la situación de la época, asegurando que, aunque en un principio la mayoría del público se negó a aceptar las innovaciones propuestas por los modernistas, por la simple razón de que transgredían su reducido esquema mental, finalmente, y por pura inercia, acabó acostumbrándose y aceptando esas innovaciones que con el tiempo resultaban más familiares:

La palabra Modernismo, que hoy denomina vagamente la última etapa de nuestra literatura, era entonces un dicterio complejo de toda clase de desprecios. Y no era lo peor esta enemiga natural del vulgo, contrario siempre a toda novedad. A las buenas gentes se les desquiciaba su tinglado mental y se les complicaba cruelmente su saneado par de ideícas con que tan bien hallados estaban. Aullaron, pues, buenamente, como los perros a la luna y prorrumpieron en ineptas risotadas durante algún tiempo, y aceptaron al cabo, sin más reflexión y por instinto. En cuanto ya estuvieron un poco fanés, las vitandas novedades ${ }^{4}$.

Las novedades modernistas han sido estudiadas por distintos autores, y en este sentido, María Victoria Utrera da constancia en su artículo «Ecos del simbolismo en la métrica modernista» de dichas innovaciones:

La levedad y la evanescencia, el matiz y el equívoco, la languidez y la vaguedad son los nuevos valores, de ahí la musicalidad, que se traduce en el verso impar, y en otros aspectos formales de carácter métrico y retórico. [...] La sutileza y la suavidad son cualidades importantes frente a la rotunda solidez del verso de épocas anteriores ${ }^{5}$.

${ }^{3}$ Utrera Torremocha, María Victoria: Poéticas de la enfermedad en la literatura moderna, Madrid: Clásicos Dyckinson, 2015, p. 44.

${ }^{4}$ Machado, Manuel: La guerra..., cit., pp. 104-105.

${ }^{5}$ Utrera Torremocha, María Victoria, «Ecos del simbolismo en la métrica modernista: El verso alejandrino». Rhythmica, Revista española de Métrica Comparada, 2010, VIII, 8, p. 221. 
Respecto a la etapa modernista de Manuel Machado y otros modernistas, destaca José Cenizo Jiménez en su artículo «Alma, Manuel Machado y el Modernismo» la raigambre simbolista de este movimiento, que tendría su origen en el Romanticismo:

El Modernismo español es más simbolista que parnasiano, a excepción de Salvador Rueda. Los demás, como los Machado, se avienen mejor con esa poesía de la sutilidad, de las impresiones, de la vaguedad lírica, la rima tenue y el metro breve que Bécquer inicia para nosotros y los poetas franceses hacen madurar para todos. [...]. El Modernismo renovó, en fin, la literatura española, que recuperó el pulso perdido desde Bécquer y Rosalía, y se adentraba en la modernidad poética, usando la lengua lírica del Xx. El ejemplo de Manuel es demostrativo ${ }^{6}$.

De esta manera, aunque hoy en día tanto la crítica como los poetas coinciden en reconocer en Manuel Machado a un creador único y original, en el que convergen distintas corrientes estéticas de la época de Fin de Siglo (Noventayochismo, Romanticismo, Populismo, Decadentismo, Malditismo...), el Modernismo ocupa un lugar central en su producción literaria y, por tanto, el objeto de este artículo es analizar algunos de los rasgos y características que sitúan a Manuel Machado en esta tradición modernista y especialmente su vinculación con la renovación del soneto.

Como es sabido, el poeta sevillano inicia su etapa modernista bajo el influjo de Rubén Darío y de la tradición simbolista francesa con la publicación de Alma (1902). A esta obra hay que sumar otros libros publicados con posterioridad, como Caprichos (1905), La fiesta nacional (Rojo y negro) (1906), El mal poema (1909), Alma. Museo. Los cantares (1907) o Apolo. Teatro pictórico (1911), que, entre otros poemarios, pertenecen a los años de plenitud del autor ${ }^{7}$.

Alma se convierte en la palabra clave de la época. Todos los críticos y artistas del momento escriben sobre el alma del poeta,

${ }^{6}$ Cenizo Jiménez, José: «Alma, Manuel Machado y el Modernismo». Cauce. Revista de filología y su didáctica, 2003, 26, p. 49.

7 Romero Luque, Manuel: Las ideas poéticas de Manuel Machado. Sevilla: Diputación provincial de Sevilla, 1992, pp. 108ss. 
de las cosas, de los seres, de la naturaleza; el alma universal, contemporánea y nacional; el alma como rebelión y como liberación y, sobre todo, como vínculo entre el Ideal, la Belleza y el Arte en el inestable sistema ideológico y estético de todo el siglo xx. Tal como afirma Rafael Alarcón Sierra en el prólogo a Alma, Caprichos y El mal poema, «es precisamente todo este estado de cosas -de almas- que flota en el ambiente finisecular el que va a recoger Machado, a modo de catalizador, en el título de un poemario fundamental: Alma» ${ }^{8}$. Así, la búsqueda de una nueva estética acorde con los movimientos del alma se traduce en una original visión artística.

En pocos años desde la publicación de sus libros modernistas, Manuel Machado alcanzó la categoría de figura central del mejor Modernismo, y es precisamente en su obra Alma donde se aprecian más claramente esos aspectos modernistas que revolucionan la poesía tal y como se entendía en nuestro país hasta aquel momento.

Sin embargo, como señala Manuel Romero Luque en su libro Las ideas poéticas de Manuel Machado, «Manuel Machado, ecléctico siempre y fiel a su propia personalidad, tomó para sí (del Modernismo) aquello que más le convenía» ${ }^{9}$, idea que ratifica José Cenizo Jiménez al afirmar que «Alma es modernista, y mucho, pero no sólo modernista ${ }^{10}$.

A partir de estos planteamientos se estudiará la estrecha vinculación entre tradición e innovación en el campo de la renovación del verso y, concretamente, en el soneto, composición de larga vida literaria que es asimilado dentro del Modernismo con variaciones importantes que afectan al metro o a la distribución de las rimas. De acuerdo con Esteban Torre, el soneto consta de catorce versos endecasílabos, con rima consonante, distribuidos en dos cuartetos, con rima abrazada, y dos tercetos, con distintas posibilidades combinatorias en cuanto a la rima, existen, además, otras variantes ${ }^{11}$. En el contexto modernista

${ }^{8}$ Machado, Manuel: Alma, Caprichos y el Mal Poema. Edición de R. A. Sierra. Madrid: Castalia, 2000, p. 39.

9 Romero LuQue, Manuel: Las ideas poéticas..., cit., p. 158.

${ }^{10}$ Cenizo Jiménez, José: «Alma, Manuel Machado...», cit., p. 50.

11 «El soneto consta de catorce versos endecasílabos, con rima consonante, distribuidos en dos cuartetos y dos tercetos. Los cuartetos llevan las mismas rimas, abra- 
cobra especial relevancia el soneto con versos alejandrinos, en el que se altera a menudo el orden tradicional de rima en los cuartetos, con la presencia en éstos de rima cruzada o de rima cruzada y abrazada.

Manuel Machado considera que el Modernismo termina como tal hacia 1911, según declara en La guerra literaria. De los libros publicados hasta esa fecha, el corpus de sonetos es el siguiente: en Alma hay tres, titulados «La corte», «Oriente» y «Wagner», todos sonetos alejandrinos; diez en Caprichos: «La primavera I y II», «Rosa...», «La voz que dice...», «Kyrie Eleyson», «La buena canción», «Sé buena I y II», «Domingo» y «Oraciones a ella», donde se mezclan los sonetos alejandrinos con los sonetos clásicos de versos endecasílabos y un soneto en arte menor; ninguno en La fiesta nacional (Rojo y negro) ni en El mal poema; cinco en Museo, «Renacimiento: En un rincón de la catedral»; «Siglo de Oro: Madrid viejo»; «Un hidalgo»; «Las concepciones de Murillo» y «Jardín neoclásico», donde todos son sonetos alejandrinos excepto el segundo, escrito en endecasílabos y alejandrinos, y el penúltimo, escrito en endecasílabos; y, por último, Apolo está compuesto de veintiséis sonetos en total, aunque todos ellos dentro de las convenciones clásicas en lo que concierne al uso del endecasílabo y, en general, de la rima, en la que igualmente predomina el esquema clásico, aunque existen ocasionales recuerdos modernistas por el uso del serventesio en algunos de estos sonetos. En Apolo, publicado en 1911, Machado se aleja del soneto alejandrino modernista y de los experimentos acentuales con el verso alejandrino. Interesa señalar, en este sentido, la propensión en el uso del soneto alejandrino, que luego desciende por dar prioridad al soneto clásico en endecasílabos. En la poesía posterior a 1911 volvemos a encontrar sonetos alejandrinos, además de los clásicos sonetos escritos en versos endecasílabos.

Teniendo en cuenta la concepción de creación poética del propio artista sevillano, conviene centrarse en el estudio de la

zadas: ABBA ABBA [...] Existen otras muchas posibilidades combinatorias de los últimos seis versos del soneto; pero los ocho primeros siempre se adaptan al ya mencionado esquema [...] Tal es, desde Garcilaso hasta nuestros días, la línea seguida por el soneto español, que se atiene al modelo petrarquista» (TorRE, Esteban: Métrica española comparada. Sevilla: Universidad de Sevilla, 2000, p. 131). 
forma externa de los poemas, prestando a su vez atención a la forma interna y al fondo de las composiciones, porque según declara el propio Manuel Machado el Modernismo fue, en definitiva, una revolución formal, tanto en la forma visible del arte, el lenguaje, como en la invisible:

El Modernismo, $[\ldots]$ no fue en puridad más que una revolución literaria de carácter principalmente formal. Pero relativa no sólo a la forma externa, sino a la interna del arte. En cuanto al fondo, su característica esencial es la anarquía ${ }^{12}$.

Debe entenderse aquí la palabra «anarquía» como lo íntimo, lo individual de cada ser, lo personal, su alma. Así, tanto Manuel Machado como sus contemporáneos modernistas entendían que el «fondo» era el pilar básico de la creación literaria, y que en definitiva, se reducía al sentimiento sincero por parte del artista de los temas que elabora; mientras que con «forma interna» se referían a esa necesidad que el poeta siente de comunicar, de expresar su sentimiento, unida al trabajo interior que supone el hallazgo de la expresión precisa; y, por último, la «forma externa» resulta ser donde mejor se puede apreciar la renovación poética llevada a cabo por el Modernismo, siendo Manuel Machado, como ya se ha mencionado, uno de los abanderados en todo tipo de innovaciones de esta índole. Por esto, aunque no debemos olvidar que estos tres pilares sostienen conjuntamente cualquier creación poética y, por tanto, no pueden considerarse apartados y distantes entre sí, aquí nos vamos a centrar en el análisis de la forma externa del poema y más concretamente, en relación a la métrica o prosodia porque, según subraya el propio Manuel Machado, «respecto a la forma externa de la poesía, las innovaciones son de dos clases: retóricas y prosódicas. [...] Las retóricas importan poco. [...] En cuanto a las novedades prosódicas, ya han sido más hondas y positivas» ${ }^{13}$.

Desde esta perspectiva, se va a considerar la base métrica modernista en relación con algunos sonetos del autor y se profundizará en seis aspectos clave: el uso del alejandrino en el soneto, las dislocaciones acentuales, las palabras al final de hemistiquio

${ }_{12}$ Machado, Manuel: La guerra ..., cit., p. 122.

13 Ibid., pp. 133-134. 
de terminación aguda o esdrújula, los encabalgamientos, la rima y los juegos fónicos.

En primer lugar, es destacable que los sonetos de Alma, tanto «Oriente», como «La corte»y «Wagner», son sonetos compuestos en alejandrinos, muy distintos al soneto tradicional, tal como se ha definido más arriba. La elección del metro alejandrino revela ya la disposición del autor, que considera necesario una renovación métrica, vinculada a la tradición francesa y dariana. Como puede apreciarse en «Oriente» los alejandrinos quedan divididos en dos isostiquios por una cesura:
An-to-nioen-los- a-cen-tos / de-Cleo-pa-traen-can-ta-do
la-co-pa-deo-rool-vi-da / quees-tá-de-néc-tar-lle-na
Y-cre-yen-teen-los-sue-ños / quee-vo-ca-la-si-re-na
to-daen-los-o-jos-tie-ne / su-al-ma-de-sol-da-do
$(7+7=14)$
$(7+7=14)$
$(7+7=14)$
$(7+7=14)$
La-rei-naho-ja-tras-ho-ja, / des-ho-jan-do-sus-flo-res
en-la-co-pa-deAn-to-nio / las-de-ja-dul-ce-men-te
Y-pro-si-gue-su-cuen-to / de-ba-ta-llas-ya-mo-res
$(7+7=14)$
$(7+7=14)$
$(7+7=14)$
a-pren-di-doen-las-ma-gas / tra-di-cio-nes-deO-rien-te
De-tié-ne-se-YAn-to-nio / ve-su-co-paol-vi-da-da
Mas-po-nee-lla-la-ma-no / so-breel-bor-de-de-o-ro
$(7+7=14)$
$(7+7=14)$
y-son-ri-en-do-len-ta / ha-cia-sí-la-re-ti-ra
$(7+7=14)$
Des-pués-siem-prea-los-o-jos / del-gue-rre-roa-so-ma-da
se-lla-sus-grue-sos-la-bios / con-un-be-so-so-no-ro
$(7+7=14)$
$(7+7=14)$
y-da-la-co-paaun-sier-vo / que-la-be-be-yex-pi-ra
$(7+7=14)$

El hecho de escribir un soneto en alejandrinos obedece precisamente al deseo de renovación antes señalado. Por un lado, tal y como afirma Manuel Romero Luque, el soneto en la época era una forma caduca, estanca, que había perdido su dinamismo, y que necesitaba urgentemente una renovación, y es precisamente Manuel Machado una de las figuras más importantes en este sentido: 
El soneto era por aquellos años una forma caduca, agobiada por su propio peso y que no conseguía erguirse sobre sí misma. Los modelos repetidos habían llegado a convertirse en una triste parodia del soneto clásico por agotamiento. Sólo una renovación del concepto de la propia estrofa, ayudada por esos moldes métricos que se forjan en el Modernismo, posibilitará que Manuel Machado devuelva su tradicional energía a esta composición ${ }^{14}$.

Por otro lado, para llevar a cabo dicha renovación del soneto y teniendo presente la gran importancia que Manuel Machado concede al ritmo del poema, que debe adecuarse al fondo aunque para ello tenga que salirse de los límites prosódicos establecidos, es comprensible que haya elegido precisamente el verso alejandrino para componer su obra, ya que como explica Manuel Romero Luque:

Para Manuel Machado lo primordial es, sin duda, hallar el ritmo que se desprende de la buena poesía y no una mera repetición de determinados pies métricos [...] En poesía, la manera de obtenerlo no era otra que salir de los acentos tónicos obligatorios de cada verso y, para ello, los modernistas potenciaron el desarrollo de los acentos secundarios. [...] Así pues, es fácil deducir que su aplicación más importante se dé en el caso de los alejandrinos; en donde la existencia de una pausa medial o cesura condiciona los acentos. Es por este logro por el que sin duda el alejandrino pierde su rigidez primitiva y se convierte en el metro preferido de Manuel Machado y de todo el modernismo en general ${ }^{15}$.

El alejandrino es, pues, el verso preferido del movimiento modernista por sus muchas capacidades expresivas y por tanto no debe extrañarnos que Manuel Machado escogiese precisamente este tipo de verso para renovar el viejo soneto español. Las sílabas pierden, en ocasiones, su condición de sílabas tónicas $\mathrm{y}$, a veces, las átonas sufren un incremento acentual que favorece la variación rítmica del verso alejandrino.

En segundo lugar, por tanto, y dada la importancia que Manuel Machado y todos los modernistas en general daban al ritmo del poema, resulta interesante fijarnos en las variedad rítmica de la composición, en busca de un ritmo menos monótono, combinando

14 Romero LuQue, Manuel: Las ideas poéticas..., cit., pp. 183-184.

15 Ibid., pp. 196-197. 
versos de ritmo binario y ternario. Sirva de nuevo un fragmento del poema «Oriente» como muestra de las innovaciones rítmicas que aparecen en los distintos sonetos modernistas del poeta:

António, en lòs acéntos / de Cleopátra encantádo, $\left(2^{\mathrm{a}}, 4^{\mathrm{a}}, 6^{\mathrm{a}} / 3^{\mathrm{a}}, 6^{\mathrm{a}}\right)$ la cópa de óro olvída / que está de néctar lléna. $\left(2^{\mathrm{a}}, 4^{\mathrm{a}}, 6^{\mathrm{a}} / 2^{\mathrm{a}}, 4^{\mathrm{a}}, 6^{\mathrm{a}}\right)$ Y, creyénte en los suéños / que evóca là siréna, tóda en los ójos tiéne / su álma dè soldádo.

En este sentido, no debemos olvidar las palabras de Manuel Machado sobre esta cuestión, al reclamar la variedad rítmica en una crítica velada a la iteración del ritmo de cláusulas en el verso regular, apreciable, por ejemplo, en Espronceda y algunos otros poetas:

No concebían los prosodistas que se saliera de los acentos tónicos obligatorios [...] Y hubo, sin embargo, dos poderosísimas razones para salir de ellos. [...] La primera [...] para dar (a la poesía) expresión real y amplitud ideológica. [...] La segunda razón, y quizá la más fuerte, es la de que no entrando la poesía solamente por el oído, sino tratando de dar sensaciones a la vista y a la inteligencia, la isócrona repetición constante de los acentos acapara y distrae la atención del lector, molestándola y separándola de otras sensaciones más interesantes ${ }^{16}$.

En tercer lugar, conviene detenerse en los finales de hemistiquios y versos. En «Oriente», por ejemplo, encontramos el uso de la terminación llana en el final de verso y de hemistiquio, como se aprecia, por ejemplo, en «An-to-nioen-los-a-cén-tos (7) / deCleo-pa-traen-can-tá-do (7) $(7+7=14)$, pero ya en «Wagner» aparecen hemistiquios de final agudo y esdrújulo que se aproximan más a la estética modernista: «Un-re-loj-que-no-sé $(6+1=7)$ / don-des-ta-da-la-u-na $(7)(7+7=14) »$, donde la última palabra del primer hemistiquio es un monosílabo agudo, por lo que se añade una sílaba al cómputo del verso; o en «en-que-la-luz-penúl-ti-ma $(8-1=7)$ / de-la-Tie-rra-sea-pa-ga (7) $(7+7=14) »$, donde ocurre lo contrario, la última palabra del primer hemistiquio es esdrújula y por tanto resta una sílaba.

$\mathrm{Si}$ en «Oriente» $\mathrm{y}$ en «La corte» todas las palabras finales de hemistiquio son llanas, manteniendo así, el cómputo silábico

${ }^{16}$ Machado, Manuel: La guerra..., cit., pp. 113-114. 
tradicional, no ocurre lo mismo en «Wagner», poema que puede dar lugar a confusiones en el cómputo silábico si no se tiene en cuenta el fenómeno de la pausa medial. En este sentido, y para evitar malentendidos, son relevantes las siguientes observaciones de Esteban Torre, que ha estudiado en profundidad el alejandrino, tanto español como francés, y no ha dejado dudas sobre la naturaleza bipartita del alejandrino en nuestra poesía, de manera que el alejandrino español, mal llamado de trece sílabas, falsamente irregular, sería sólo el resultado de una escansión errónea, que no tiene en cuenta fenómenos como el de la cesura:

Evidentemente, tanto el llamado alejandrino a la francesa como el ternario no son más que el resultado de una lectura inconsecuente con las convenciones de la métrica española. El alejandrino castellano es siempre un verso compuesto de « $7+7 》$ sílabas, esto es, un tetradecasílabo con cesura entre dos hemistiquios heptasilábicos ${ }^{17}$.

En los sonetos alejandrinos de Manuel Machado la tendencia a la experimentación métrica de carácter simbolista y modernista es más evidente en Caprichos y va disminuyendo progresivamente, respecto del uso del mal llamado alejandrino tripartito, en los sonetos publicados en libros posteriores. Si en el poema «Adelfos» de Alma hay ya ejemplos de desplazamientos e incrementos acentuales en los hemistiquios de los versos alejandrinos ${ }^{18}$, esta tendencia a la experimentación con el alejandrino se cumple, sobre todo, en los sonetos de Caprichos, que ofrecen casos de sílabas átonas a final de verso o hemistiquio con incremento acentual y encabalgamiento fuerte e incluso ejemplos de división de palabra entre hemistiquios por el efecto de la cesura, produciéndose el encabalgamiento léxico o tmesis. Al caso del verso «La Caridad, La Caridad, La Caridad» («La Caridad, La Cà- / ridad, La Caridad») ${ }^{19}$, del soneto titulado «Kyrie Eleyson», se suman otros. Así, encontramos en este mismo soneto incrementos acentuales en sílabas átonas que en el contexto métrico alejandrino adquieren un valor tónico, además de otro ejemplo

\footnotetext{
17 Torre, Esteban: Métrica española..., cit., p. 88.

${ }^{18}$ Manuel Romero Luque menciona el verso «En mi alma, hermana de la tarde, no hay contornos» («En mi alma, hermana dè / la tarde, no hay contornos»). Véase Romero LuQue, Manuel: Las ideas poéticas..., cit., p. 198.

${ }^{19}$ Ibid.
} 
de tmesis: «y tu corona de espinas, y tu diestra» («y tu corona dè / espinas y tu diestra»), «horadada por el clavo de la impiedad» («horadada por èl / clavo de la impiedad»), «Y que un paisaje matinal y que una buena» («Y que un paisaje mà- / tinal y que una buena»). Esta modulación sobre el verso alejandrino reaparece en otros sonetos de Caprichos. En el soneto «Sé buena, I»: «la ternura de tu corazón, sin las hueras» («la ternura de tù / corazón, sin las hueras»), «flores de trapo de la retórica vana» («flores de trapo dè / la retórica vana»), «de una pasión conceptuosa y sus maneras...» («de una pasión concèp- / tuosa y sus maneras...»); en el soneto «Sé buena, II»: «Y, en una dulce convalecencia, una mañana» ( $\mathrm{Y}$, en una dulce conva- / lecencia, una mañana»), «movido como por una alocada risa» («movido como pòr / una alocada risa»). En libros posteriores este tipo de alejandrino es menos frecuente. Se encuentra ocasionalmente en algún soneto de $M$ useo, como el titulado «Un hidalgo»: «tiene derecho a descansar y estar ahora» («tiene derecho a dès- / cansar y estar ahora»).

De acuerdo con lo anterior, y en cuarto lugar, son frecuentes, por tanto, los encabalgamientos entre hemistiquios, algunos de marcada influencia modernista. En «Oriente» existe un gran número de encabalgamientos mediales, es decir, entre hemistiquios, todos ellos de carácter suave. Esta abundancia de encabalgamientos mediales, aunque sean de carácter suave, crea una tensión en el poema que dinamiza el ritmo de toda la composición. Como afirma José Domínguez Caparrós en su libro de Elementos de métrica española, "si se hace la pausa versal, se rompe un grupo sólidamente unido; si no se hace la pausa versal, se rompe la unidad del verso. Esta tensión es la fuente de los valores estilísticos del encabalgamiento» ${ }^{20}$. Especialmente en Caprichos es evidente que el encabalgamiento adquiere un especial protagonismo por la presencia de encabalgamientos léxicos y sintagmáticos, según se ha expuesto ya.

En quinto lugar, en relación a la rima, Manuel Romero Luque afirma que «para el poeta sevillano la rima tiene un valor muy importante, de ahí la visión negativa que se desprende cuando

${ }^{20}$ Domínguez Caparrós, José: Elementos de métrica española, Valencia: Tirant lo Blanch, 2005, p. 45. 
un poema es «de rima consabida», es decir, un ripio; porque esto es lo mismo que un poema sin personalidad ${ }^{21}$.

En general, el soneto alejandrino de Manuel Machado tiene pocas innovaciones respecto al orden de la rima. En «Oriente» se aprecian algunas. Así, aunque el primer cuarteto sigue el esquema de rima abrazada consonante tradicional (ABBA) y los tercetos riman siguiendo el esquema clásico (EFG) (EFG), el segundo cuarteto introduce la rima cruzada (CDCD). Hay que recordar que el cuarteto de rima cruzada o serventesio es frecuente en el soneto modernista. Igualmente, la combinación de serventesios en un mismo poema es habitual en el Modernismo y se asocia con la estética francesa. En este sentido, poemas como «Oriente» dan muestras de este gusto por la rima cruzada en los cuartetos. No hay grandes innovaciones en cuanto a la rima del poema como cabría esperar de una composición modernista, pero sí que se aprecian ciertas rimas asonantes no regulares entre hemistiquios próximos (é-o, é-e, ó-o, á-a), que se señalan en cursiva:

Antonio, en los acentos de Cleopatra encantado, A la copa de oro olvida que está de néctar llena. B B $\mathrm{Y}$, creyente en los sueños que evoca la sirena, B toda en los ojos tiene su alma de soldado. A

La reina, hoja tras hoja, deshojando sus flores, C en la copa de Antonio las deja dulcemente... $\quad \mathrm{D}$ Y prosigue su cuento de batallas y amores, C aprendido en las magas tradiciones de Oriente... $\quad$ D

Detiénese... Y Antonio ve su copa olvidad $a . . . \quad$ E Mas pone ella la mano sobre el borde de oro, F $\mathrm{y}$, sonriendo, lenta hacia sí la retira...

A
B
B
A
C
D
C
D
E
F
G

Después, siempre a los ojos del guerrero asomad $a, \mathrm{E}$ sella sus gruesos labios con un beso sonoro... F F y da la copa a un siervo, que la bebe y expira... G

En Caprichos se observa, en cuanto a la rima, una intensificación en la manera modernista. Así, en los sonetos alejandrinos o de mezcla entre alejandrinos y endecasílabos de Caprichos ${ }^{21}$ Romero LuQue, Manuel: Las ideas poéticas..., cit., p. 190. 
es claro el predominio de los cuartetos de rima cruzada -o serventesios-, o la mezcla de cuarteto y serventesio en un mismo soneto. Esta tendencia se atenúa en Museo, libro en el que, si bien predomina el uso del alejandrino en los sonetos, es apreciable ya el carácter algo más conservador en lo que respecta a la rima. A este respecto, solamente uno de los cinco sonetos de Museo tiene rima que combina el esquema cruzado y abrazado en los cuartetos, siendo los cuartetos de los cuatro sonetos restantes de rima abrazada. En el caso de los tercetos, la ordenación suele responder, en general, tanto para sonetos clásicos como para sonetos alejandrinos, al esquema CDE CDE, aunque existen otras variantes.

En sexto lugar, en este análisis de la forma externa, es necesario destacar la musicalidad del poema, lograda con alteraciones y uso de sonidos y de rimas internas, algunas ya indicadas en «Oriente». No podía faltar la mención al penúltimo verso de este soneto: «sella sus gruesos labios con un beso sonoro», donde encontramos una intensa aliteración que muestra el esmerado cuidado formal del poeta. A través de ese sonido producido por la repetición de la sibilante - por supuesto, unida al tema del poema, la historia de Antonio y Cleopatra-, se transmite una sensación inexplicable que apela directamente a nuestros sentidos. Este juego sensorial es propio de Manuel Machado y de los modernistas en general, que gustan del empleo de sinestesias y otros recursos para incentivar los sentidos y la imaginación, en busca de la sugerencia.

Sin embargo, tal y como nos recuerda Manuel Romero Luque, la revolución literaria no radica en unas simples innovaciones métricas, sino que es absoluta. Hay una importante renovación de la forma externa, como ya hemos visto, pero también se producen cambios en cuanto a la forma interna, es decir, en cuanto a la manera del artista de entender y crear poesía:

Machado limita el Modernismo al ámbito literario. Pero dentro de este ámbito, la revolución es total, pues afecta «no sólo a la forma externa, sino a la interna del arte», es decir, no sólo cambiará el lenguaje expresivo, sino también la manera de pensar y concebir la poesía; de ahí que suponga la creación de una poética nueva y completa ${ }^{22}$.

$\overline{{ }^{22}}$ Ibid., p.66. 
En este sentido, en relación a la «forma interna» del poema, es decir, a la manera que tiene el autor de percibir y concebir la poesía, hay que aclarar que, al igual que muchos de sus compatriotas, Manuel vivía asfixiado en la agitada época de Fin de Siglo y, en consonancia con las ideas modernistas del momento, vuelve la mirada hacia al exotismo imperante del momento. Recordemos que, como dice María Victoria Utrera, «la decepción de la realidad externa está en directa conexión con la necesidad de huida del artista $\rangle^{23}$. Sin embargo, Manuel Machado no tiene una actitud descomprometida ante la vida, sino que, por el contrario, afectado por la situación que le ha tocado vivir, se rebela contra la realidad, aunque de manera opuesta a la Generación del 98, con la que también comparte algunos rasgos, es decir, volviendo la mirada hacia mundos más placenteros, como ya hicieron los románticos en su momento. Así, el artista sevillano concibe la poesía como un arma con la que luchar contra el ambiente de la época, según expresa claramente en una de sus declaraciones ${ }^{24}$.

La libertad que Manuel Machado propugnaba para la poesía, cuya forma externa es manifestación de la interna, es, por tanto, igualmente necesaria para el fondo. La verdadera renovación artística se produce cuando el poeta consigue la perfecta cohesión entre el fondo y la forma, interna y externa, dando a su obra una perfecta naturalidad:

consecuencia de lo sincero de un escritor es la naturalidad de la obra en el fondo y aun en la forma. Esa difícil y encantadora naturalidad que da a las creaciones del arte la divina gracia de las plantas que crecen en el aire libre, bajo la luz inimitable ${ }^{25}$.

Una vez llevado a cabo este análisis de las innovaciones en el lenguaje poético de Manuel Machado en relación con la

\footnotetext{
${ }^{23}$ Utrera Torremocha, María Victoria, Poéticas de la enfermedad..., cit., p.22.

24 «Yo creo que la única política patriótica consiste en hacer cada uno lo suyo lo mejor que pueda. Yo hago versos y no otra cosa. Y cuando algún furioso militante me excita a tomar parte en alguna labor política o sociológica, suelo responderle como Guerrita a aquel otro torero, que le pedía la punta de su capote para lancear al alimón: -Toree usted con el suyo, que el mío es de seda.» (MACHADO, Manuel: La guerra..., cit., p. 117.)

25 Ibid., p. 153.
} 
revolución modernista, se puede comprobar que sin estas imprescindibles novedades no hubiese sido posible una expresión tan directa y sugerente entre el poeta y sus lectores, que adquiere unos matices y una fuerza de transmisión hasta entonces nunca vista.

\section{Bibliografía utilizada}

CENIZO JIMÉNEZ, José: «Alma, Manuel Machado y el Modernismo». Cauce. Revista de filología y su didáctica, 2003, 26, pp. 47-65.

DOMÍNGUEZ CAPARRÓS, José: Elementos de métrica española. Valencia: Tirant lo Blanch, 2005.

MACHADO, Manuel: Alma, Caprichos y el Mal Poema. Edición de R. A. Sierra. Madrid: Castalia, 2000.

-: La guerra literaria. Madrid: Narcea, 1981.

ROMERO LUQUE, Manuel: Las ideas poéticas de Manuel Machado. Sevilla: Diputación provincial de Sevilla, 1992.

TORRE, Esteban: Métrica española comparada. Sevilla: Universidad de Sevilla, 2000.

UTRERA TORREMOCHA, María Victoria: «Ecos del simbolismo en la métrica modernista: El verso alejandrino». Rhythmica, Revista española de Métrica Comparada, 2010, VIII, 8, pp. 219-234.

- Poética de la enfermedad en la literatura moderna. Madrid: Clásicos Dyckinson, 2015. 